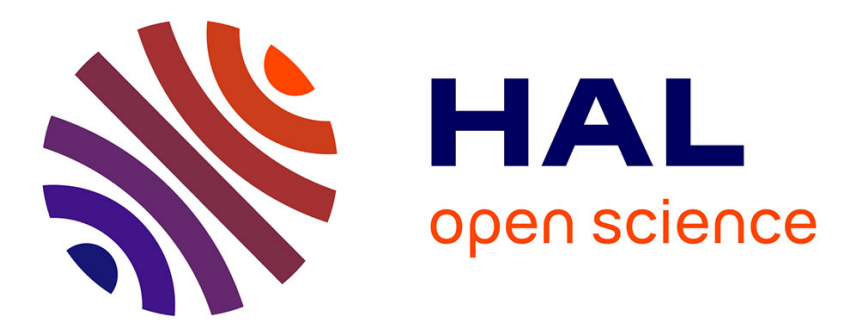

\title{
Speed-sensorless control of switched-reluctance motors with uncertain payload
}

Antonio Loria, G. Espinosa-Perez, Erik Chumacero, Missie Aguado-Rojas

\section{To cite this version:}

Antonio Loria, G. Espinosa-Perez, Erik Chumacero, Missie Aguado-Rojas. Speed-sensorless control of switched-reluctance motors with uncertain payload. 2013 IEEE American Control Conference ACC, Jun 2013, Washington DC, United States. pp.3437-3442, 10.1109/acc.2013.6580362 . hal-00831454

\section{HAL Id: hal-00831454 https://hal.science/hal-00831454}

Submitted on 7 Jun 2013

HAL is a multi-disciplinary open access archive for the deposit and dissemination of scientific research documents, whether they are published or not. The documents may come from teaching and research institutions in France or abroad, or from public or private research centers.
L'archive ouverte pluridisciplinaire HAL, est destinée au dépôt et à la diffusion de documents scientifiques de niveau recherche, publiés ou non, émanant des établissements d'enseignement et de recherche français ou étrangers, des laboratoires publics ou privés. 


\title{
Speed-sensorless control of switched-reluctance motors with uncertain payload
}

\author{
Antonio Loría Gerardo Espinosa-Pérez Erik Chumacero Missie Aguado-Rojas
}

\begin{abstract}
We present a controller for switched-reluctance motors without velocity measurements and provide, to the best of our knowledge, the first result establishing global exponential stability. Our controller is composed of an "internal" tracking control loop for the stator dynamics and an "external" control loop based on the so-called $\mathbf{P I}^{2} \mathrm{D}$ controller. The latter consists in a linear proportional derivative controller in which the measurement of velocities is replaced by approximate derivatives. Furthermore, a double integrator is added to compensate for the load uncertainty. We illustrate our theoretical findings with numerical simulations.
\end{abstract}

\section{INTRODUCTION}

In spite of the bulk of literature on control of electrical machines, a number of problems remain open. This is in part due to the specificities of each motor, which hardly allow for a universal controller. Among open complex control challenges in the field of electrical machines the necessity of eliminating the use of sensors for the mechanical variables (position and/or speed) is of special interest. This problem, well-known as sensorless control, is beyond the theoretical interest entailed by its difficulty -the requirement of not using mechanical sensors has its roots both in practical limitations and economic reasons. On one hand, mechanical sensors exhibit undesirable behaviors in several scenarios such as high-noise sensitivity and reduced reliability. On the other, economic factors cannot be overestimated -the operation and implementation of this type of measurement devices may drastically increase the cost of a given setup.

Switched-reluctance drives are typically highly reliable and produce high torque at low speeds however, they are highly nonlinear electromechanical machines since the generated torque is a nonlinear function of stator currents and rotor positions. The control design relies on the ability to separate the machine model into its electric and mechanical components. Torque generation is achieved by following the "torque-sharing" approach of [1] with the aim of reducing the ripple in the mechanical variables that appears due to the electric commutation.

The scenario is considerably different for electric machines which admit the Blondel-Park transformation, widely used in control design for induction motors, permanentmagnet synchronous motors, etc. For instance, in [2] the

\footnotetext{
A. Loría is with CNRS. E. Chumacero is with Univ. Paris Sud. Address: LSS-UPELEC, 91192 Gif-sur-Yvette, France. E-mail: antonio.lorialss.supelec.fr, erik. chumacero@lss.supelec.fr

G. Espinosa-Pérez and M. Aguado-Rojas are with FI UNAM, Edificio de Posgrado, 04510 México D.F., MEXICO. gerardoe@servidor.unam.mx.
}

authors propose a controller for induction motors without speed measurements. The controller is based on indirect field orientation and relies on a high-gain speed estimator. Local asymptotic stability is established even in the case of unknown load. In [3] the authors present a speed sensorless controller for induction motors which guarantees global asymptotic stability. This is extended to the case of full sensorless control (no position measurements) in [4] however, the result holds by restricting initial conditions to a specified domain of attraction.

In contrast to this, the fact that the model of switchedreluctance motors does not admit such handy change of coordinates, imposes a considerable challenge to theoretical validation. Articles containing a rigorous stability analysis, especially in a sensorless context, are rare. The main result in [5] establishes global asymptotic stability for a passivitybased controller in the case of unknown load however, it uses both mechanical variables, angular velocity and position measurements. On the other hand, a proportional-derivativebased controller is proposed in [1] but relying on the knowledge of the torque load. Relatively efficient but "solely" experimentally-validated approaches have been proposed in [6], [7], [8], [9], [10], to mention only a few. Indeed, these controllers constitute ad-hoc engineered solutions to the sensorless problem and none of these articles includes a study of stability.

In this paper, we exploit the physical characteristics of the nonlinear model and propose a relatively simple controller which consists in two control loops. An "internal" loop which steers the stator currents to a desired "reference" that may be regarded as a virtual control input, closing an external loop with the mechanical dynamics. The external control input has the structure of a proportional integral derivative controller. Specifically, we use the so-called $\mathrm{PI}^{2} \mathrm{D}$ controller originally proposed in [11] for robot manipulators. The control law consists in a term proportional to the angular position error, a derivative term which is proportional to an approximate derivative of the angular positions and two integrators. One integral term of the angular position errors and one integral correction term which depends on the approximate differentiation of the angular velocities. We show via Lyapunov's direct method, global exponential stability of the origin of the closed-loop.

The rest of the paper is organized as follows. In the next section we present the motor model and explain the sharingfunctions implementation approach. In Section III we present our main result. Simulations that illustrate our theoretical findings are provided in Section IV. 


\section{THE MOTOR MODEL}

After experimental evidence, it is well accepted that the three stator phases of a switched-reluctance motor may be assumed to be magnetically decoupled i.e., the mutual inductance among stator phases is negligible. Then, a general three-phase dynamic model is given by

$$
\begin{aligned}
\dot{\psi}_{j}(\theta, i)+R i_{j} & =u_{j}, \quad j=1,2,3 ; \\
J \dot{\omega} & =T_{e}(\theta, i)-T_{L}(\theta, \omega)
\end{aligned}
$$

where: for each phase $j, u_{j}$ is the voltage applied to the stator terminals, $i_{j}$ is the stator current, $\psi_{j}$ is the flux linkage; $R$ is the stator winding resistance, $\omega$ is the angular velocity, $\theta$ is the angular rotor position hence, $\omega=\dot{\theta} ; T_{L}$ is the load torque (assumed unknown), $J$ is the total rotor inertia and $T_{e}$ is the mechanical torque of electrical origin, which depends on both the angular rotor position and on all the stator currents $i=\left[i_{1}, i_{2}, i_{3}\right]^{\top}$.

Assuming that the machine operates at low current levels, it is common practice to express the inductance of each phase as a strictly positive Fourier series truncated at the first harmonic i.e.,

$$
L_{j}(\theta)=l_{0}-l_{1} \cos \left(N_{r} \theta-(j-1) \frac{2 \pi}{3}\right)
$$

where $l_{0}>l_{1}>0$ and, for further development, we remark that there exist constants $l_{m}, l_{M}$ and $k_{M}$ such that for all $\theta \in[-\pi, \pi]$ and $j \in\{1,2,3\}$,

$$
0<l_{m} \leq\left|L_{j}(\theta)\right| \leq l_{M}, \quad\left|K_{j}(\theta)\right| \leq k_{M} .
$$

The dynamic model of the motor becomes

$$
\begin{aligned}
u_{j} & =L_{j}(\theta) \frac{d i_{j}}{d t}+K_{j}(\theta) \omega i_{j}+R i_{j} \\
J \dot{\omega} & =T_{e}(\theta, i)-T_{L}(\theta, \omega)
\end{aligned}
$$

where

$$
K_{j}(\theta)=\frac{\partial L_{j}}{\partial \theta}=N_{r} l_{1} \sin \left(N_{r} \theta-(j-1) \frac{2 \pi}{3}\right)
$$

corresponds to the phase-inductance variation relative to the rotor angular position. Correspondingly, the mechanical torque for each phase is given by

$$
T_{j}=\frac{1}{2} K_{j}(\theta) i_{j}^{2}
$$

and, considering that the stator windings are decoupled, the mechanical torque of electrical origin corresponds to the sum of torques produced by each of the three phases,

$$
T_{e}(\theta, i)=\sum_{j=1}^{3} T_{j}\left(\theta, i_{j}\right) .
$$

The model (3) is adopted in both the electrical-machines and the control research communities $-c f$. [12]; a more detailed description is available in [5].

The control goal is to design a dynamic controller for (3) with output $u=\left[\begin{array}{lll}u_{1} & u_{2} & u_{3}\end{array}\right]^{\top}$ depending on the stator currents and rotor angular positions, such that $\omega(t)$ tracks any bounded smooth desired trajectory $\omega^{*}$. Hence, the purpose is to avoid the use of speed sensors in the control scheme.

\section{A. Torque delivery}

Generally speaking, the control design starts with a given desired reference $\omega^{*}$. Then, a desired control input $T_{d}$ is designed for the mechanical equation (3b) to steer $\omega \rightarrow \omega^{*}$. The control $T_{d}$ must be implemented "through" the mechanical torque $T_{e}$ that is, we define the reference mechanical torque $T_{e}^{*}$ which satisfies

$$
T_{e}^{*}\left(\theta, i^{*}\right)=\frac{1}{2}\left(K_{1}(\theta) x_{1}^{* 2}+K_{2}(\theta) x_{2}^{* 2}+K_{3}(\theta) x_{3}^{* 2}\right)
$$

where $x_{j}^{*}$ is a current reference trajectory for each phase, which must be defined as a solution to

$$
\frac{T_{e}^{*}}{J}=T_{d}
$$

for any given $T_{d}$. Hence, provided that $T_{e}=T_{e}^{*}$ the desired control torque $T_{d}$ acts upon the mechanical equation to drive $\omega \rightarrow \omega^{*}$. By ensuring an accurate current tracking that is, $i \rightarrow i^{*}$ it is guaranteed that $T_{e} \rightarrow T_{e}^{*}$ and consequently, that $\omega \rightarrow \omega^{*}$.

In order to solve (4) for $x_{j}^{*}$, we exploit the physics of the switched reluctance motor. In view of the fact that the torque sign is only determined by the variation of the inductance and using ideas reported in [13] and [1] we introduce the following current-switching policy. Define the sets

$$
\begin{aligned}
& \Theta_{j}^{+}=\left\{\theta \in[-\pi, \pi]: K_{j}(\theta) \geq 0\right\} \\
& \Theta_{j}^{-}=\left\{\theta \in[-\pi, \pi]: K_{j}(\theta)<0\right\}
\end{aligned}
$$

where the superscripts ${ }^{+}$and ${ }^{-}$stand for required positive and negative torque respectively. Accordingly, let us introduce

$$
\begin{aligned}
& m_{j}^{+}(\theta)>0, \sum_{j=1}^{3} m_{j}^{+}(\theta)=1 \quad \forall \theta \in \Theta^{+}, \\
& m_{j}^{-}(\theta)>0, \sum_{j=1}^{3} m_{j}^{-}(\theta)=1 \quad \forall \theta \in \Theta^{-}
\end{aligned}
$$

and given $T_{d}$, define

$$
m_{j}(\theta)=\left\{\begin{array}{lll}
m_{j}^{+}(\theta) & \text { if } \quad T_{d} \geq 0, \\
m_{j}^{-}(\theta) & \text { if } \quad T_{d}<0 .
\end{array}\right.
$$

Then, the reference currents for $j \in\{1,2,3\}$ are given by

$$
x_{j}^{*}= \begin{cases}{\left[\frac{2 J m_{j}(\theta) T_{d}}{K_{j}(\theta)}\right]^{1 / 2}} & \text { if } N_{r} \theta \neq(j-1) \frac{2 \pi}{3} \\ 0 & \text { otherwise. }\end{cases}
$$

The definition of $m_{j}$ ensures that $x_{j}^{*}$ exists for any $\theta$ and $T_{d}$ $-c f$. [5]. That is, depending on the current phase of the motor, the function $m_{j}(\theta)$ ensures that the respective signs of the numerator and of the denominator in the previous expression are equal for at least one $j \in\{1,2,3\}$ and the denominator $\sin \left(N_{r} \theta-(j-1) \frac{2 \pi}{3}\right) \neq 0$ and by construction, we have

$$
T_{d}=m_{1}(\theta) T_{d}+m_{2}(\theta) T_{d}+m_{3}(\theta) T_{d} .
$$

Roughly speaking, the virtual control $T_{d}$ is induced into the mechanical dynamics through a different reference, depending on the current phase. 


\section{MAIN RESULT}

We establish global exponential stability of the closedloop system under the action of a controller of ProportionalIntegral-Derivative type. It is reminiscent of the ProportionalDerivative plus load compensation presented in [13] where the sharing-functions approach was proposed. However, we assume that the load torque is unknown and that velocity is not measured, so we use the so-called $\mathrm{PI}^{2} \mathrm{D}$ controller originally proposed in [11]. Our main result extends previous work using both mechanical velocity and position measurements as in [5] as well as [1]. In contrast with available sensorless ad hoc controllers proposed without theoretical foundation, we establish global exponential stability hence robustness with respect to external disturbances, neglected dynamics, etc.

For convenience, we use the variable $x$ to denote the stator currents and re-write the dynamic model in a compact form. That is, let $x=i \in \mathbb{R}^{3}$ hence (3a) becomes

$$
L(\theta) \dot{x}+K(\theta) \omega x+R x=u
$$

where $L(\theta)=\operatorname{diag}\left\{L_{j}(\theta)\right\}$ and $K(\theta)=\operatorname{diag}\left\{K_{j}(\theta)\right\}$. Define also, the tracking errors $e_{x}=x-x^{*}, e_{w}=\omega-\omega^{*}$ and $e_{\theta}=\theta-\theta^{*}$ where $^{1}$

$$
\theta^{*}(t)=\int_{0}^{t} \omega^{*}(s) d s, \quad \theta^{*}(0)=\theta_{0}^{*} \in[-\pi, \pi] .
$$

Then, the mechanical equation (3b) may be equivalently written as

$$
\dot{e}_{w}=T_{d}-\frac{T_{L}}{J}+\frac{T_{e}}{J}-\frac{T_{e}^{*}}{J}-\dot{\omega}^{*} .
$$

Since $T_{e}$ and $T_{e}^{*}$ are quadratic functions, uniformly bounded in $\theta$, and taking account (2), we have

$$
\left|T_{e}-T_{e}^{*}\right| \leq \frac{k_{M}}{2}\left[\left|e_{x}\right|^{2}+2\left|x^{*}\right|\left|e_{x}\right|\right] .
$$

Consider the controller given by

$$
\begin{aligned}
u & =L(\theta) \dot{x}^{*}+K(\theta) \omega^{*} x+R x^{*}-k_{p x} e_{x} \\
T_{d} & =-k_{p} e_{\theta}-k_{d} \vartheta+\nu+\dot{\omega}^{*} \\
\dot{\nu} & =-k_{i}\left(e_{\theta}-\vartheta\right) \\
\dot{q}_{c} & =-a\left(q_{c}+b e_{\theta}\right) \\
\vartheta & =q_{c}+b e_{\theta}
\end{aligned}
$$

where $k_{p}, k_{i}, k_{d}, a, b>0$ and $k_{p x}$ is a positive strictly increasing function of the measured states, to be defined. Equations (10b)-(10e) correspond to the so-called $\mathrm{PI}^{2} \mathrm{D}$ controller originally introduced in [11] for robot manipulators.

We note that the column vector $\dot{x}^{*}=\left[\dot{x}_{1}^{*} \dot{x}_{2}^{*} \dot{x}_{3}^{*}\right]^{\top}$ depends on the unmeasurable error $e_{\omega}$, that is, the reference derivative is given by

$$
\dot{x}_{j}^{*}= \begin{cases}\alpha_{j}(\cdot)\left[\rho_{j}(\cdot)+\delta_{j}(\cdot) e_{\omega}\right] & \text { if } \theta \neq(j-1) \frac{2 \pi}{3} \\ 0 & \text { otherwise }\end{cases}
$$

\footnotetext{
${ }^{1}$ Since the variable to be controlled is $\omega$, the initial value of $\theta^{*}(t)$ is innocuous.
}

where

$$
\begin{aligned}
\alpha_{j}(\cdot) & =\frac{1}{2}\left(\frac{2 J m_{j} T_{d}}{K_{j}}\right)^{-\frac{1}{2}} \\
\rho_{j}(\cdot) & =\frac{J}{K_{j}}\left(\beta_{j}(\cdot)+m_{j}^{\prime} \omega^{*} T_{d}\right) \\
\beta_{j}(\cdot) & =m_{j}\left(k_{i d} \vartheta-k_{i} e_{\theta}+\ddot{\omega}^{*}-\frac{T_{d} K_{j}^{\prime} \omega^{*}}{K_{j}}\right), \\
\delta_{j}(\cdot) & =\frac{J}{K_{j}}\left(m_{j}^{\prime} T_{d}-m_{j}\left(k_{p d}+\frac{T_{d} K_{j}^{\prime}}{K_{j}}\right)\right),
\end{aligned}
$$

$k_{i d}=k_{i}+a k_{d}, k_{p d}=k_{p}+b k_{d}, m_{j}^{\prime}=\frac{\partial m_{j}}{\partial \theta}$ and $K_{j}^{\prime}=\frac{\partial K_{j}}{\partial \theta}$; note that $m_{j}, K_{j}$ are both dependent on $\theta$. Therefore, only the terms $\alpha(\cdot)=\operatorname{diag}\left\{\alpha_{j}(\cdot)\right\}$ and $\rho(\cdot)=$ $\left[\rho_{1}(\cdot), \rho_{2}(\cdot), \rho_{3}(\cdot)\right]^{\top}$ are used in the control law, that is,

$$
u=L(\theta) \alpha(\cdot) \rho(\cdot)+K(\theta) \omega^{*} x+R x^{*}-k_{p x} e_{x} .
$$

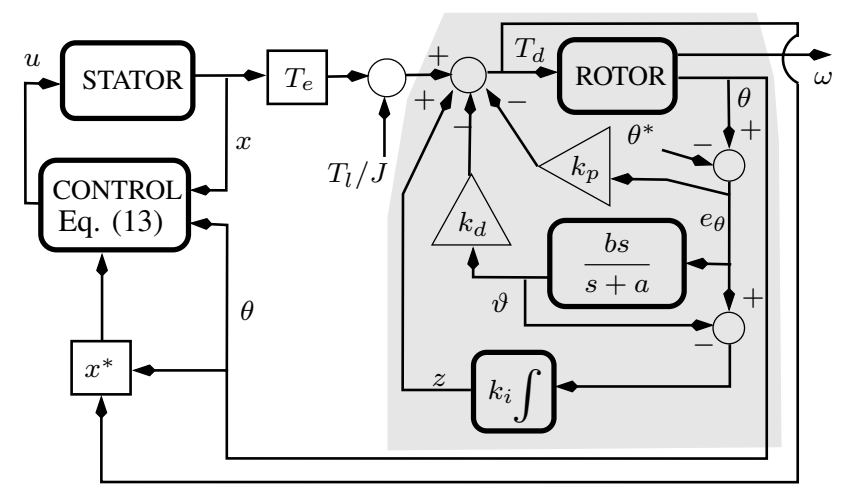

Fig. 1. The closed-loop system consists in two nested loops; an inner loop composed of the rotor dynamics and the $\mathrm{PI}^{2} \mathrm{D}$ controller and an outer loop which is closed via the actual controls (input voltages $u$ ) as well as position and currents measurements.

Proposition 1 Define $\Delta=\alpha \delta$, with $\delta=\left[\begin{array}{lll}\delta_{1} & \delta_{2} & \delta_{3}\end{array}\right]^{\top}$, then the origin of the closed-loop system composed by the motor equations (3) and the controller (6), (13), (10b) and (10c), is globally exponentially stable if

$$
\begin{aligned}
& k_{d}>\frac{3 b}{b-1}\left[\frac{k_{M}}{l_{m}}+1\right], k_{d}<\frac{3 a}{2} \frac{\lambda}{\lambda+1} \\
& b \geq 2 a+1, k_{p}^{\prime}>k_{d} \\
& k_{p x}:=k_{p x}^{\prime}+\mathcal{O}\left(|x|^{2}\right)+\mathcal{O}\left(|\Delta|^{2}\right)+\mathcal{O}\left(\left|e_{x}\right|^{2}\right)+\mathcal{O}\left(\left|x^{*}\right|^{2}\right) .
\end{aligned}
$$

An explicit expression for $k_{p x}$ is given in the proof of Proposition 1, which is based on Lyapunov's direct method and a small-gain argument. We start by writing the closedloop system. Let $\varepsilon>k_{i}$ and define

$$
z=\nu-\frac{T_{L}}{J}-\frac{k_{i}}{\varepsilon} e_{\theta} .
$$

Then, define $k_{p}^{\prime}:=k_{p}-k_{i} / \varepsilon>0$. Using (10b) and (17) in (8) and differentiating on both sides of (10e) and (17) we 
obtain

$$
\begin{aligned}
\dot{e}_{\theta} & =e_{w} \\
\dot{e}_{w} & =-k_{d} \vartheta-k_{p}^{\prime} e_{\theta}+z+\frac{1}{J}\left(T_{e}-T_{e}^{*}\right) \\
\dot{\vartheta} & =-a \vartheta+b e_{w} \\
\dot{z} & =-k_{i}\left(e_{\theta}-\vartheta\right)-\frac{k_{i}}{\varepsilon} e_{w} .
\end{aligned}
$$

Furthermore, substituting (13) in (7) we obtain

$$
\dot{e}_{x}=L^{-1}\left[-\left(R+k_{p x}\right) e_{x}-(K x(t)+L \Delta) e_{\omega}\right] .
$$

The rest of the proof relies on the following two statements which roughly speaking, allow to apply a small-gain argument.

Claim 1 The rotor dynamics (18) with state $\zeta_{1}=$ $\left[e_{\theta} e_{\omega} \vartheta z\right]^{\top}$, is input-to-state stable with input $e_{x}$.

Claim 2 If $k_{p x}$ is chosen as in Proposition 1 the stator dynamics (19) is input-to-state stable with input $e_{w}$.

Proof of Claim 1. We start by re-writing (18) as

$$
\left[\begin{array}{c}
\dot{e}_{\theta} \\
\dot{e}_{\omega} \\
\dot{\vartheta} \\
\dot{z}
\end{array}\right]=\underbrace{\left[\begin{array}{cccc}
0 & 1 & 0 & 0 \\
-k_{p}^{\prime} & 0 & -k_{d} & 1 \\
0 & b & -a & 0 \\
-k_{i} & -k_{i} / \varepsilon & k_{i} & 0
\end{array}\right]}_{A} \underbrace{\left[\begin{array}{c}
e_{\theta} \\
e_{\omega} \\
\vartheta \\
z
\end{array}\right]}_{\zeta_{1}}+\underbrace{\left[\begin{array}{c}
0 \\
1 / J \\
0 \\
0
\end{array}\right]}_{B}\left(T_{e}-T_{e}^{*}\right)
$$

Let $\varepsilon_{1}=\varepsilon$ and $\varepsilon_{2}$ be two "small" positive numbers and consider the Lyapunov function $V_{1}\left(\zeta_{1}\right)=\frac{1}{2} \zeta_{1}^{\top} P \zeta_{1}$ with

$$
P=\left[\begin{array}{cccc}
k_{p}^{\prime} & \varepsilon_{1} & 0 & 0 \\
\varepsilon_{1} & 1 & -\varepsilon_{1} & -\varepsilon_{2} \\
0 & -\varepsilon_{1} & k_{d} / b & 0 \\
0 & -\varepsilon_{2} & 0 & \varepsilon_{1} / k_{i}
\end{array}\right] .
$$

This matrix is strictly diagonal-dominant if

$$
\begin{array}{rl}
k_{p}^{\prime}>\varepsilon_{1} & 1>2 \varepsilon_{1}+\varepsilon_{2} \\
\frac{k_{d}}{b}>\varepsilon_{1} & \frac{\varepsilon_{1}}{k_{i}}>\varepsilon_{2}
\end{array}
$$

which hold for sufficiently small $k_{i}, \varepsilon_{1}$ and $\varepsilon_{2}$. Since $P$ is also symmetric, it is positive definite. Also, we have $P A=$

$$
\left[\begin{array}{cccc}
-\varepsilon_{1} k_{p}^{\prime} & k_{p}^{\prime} & -\varepsilon_{1} k_{d} & \varepsilon_{1} \\
-k_{p}^{\prime}+\varepsilon_{2} k_{i} & \varepsilon_{1}(1-b)+\frac{\varepsilon_{2} k_{i}}{\varepsilon_{1}} & -k_{d}+\varepsilon_{1} a-\varepsilon_{2} k_{i} & 1 \\
\varepsilon_{1} k_{p}^{\prime} & k_{d} & k_{d}\left(\varepsilon_{1}-\frac{a}{b}\right) & -\varepsilon_{1} \\
\varepsilon_{2} k_{p}^{\prime}-\varepsilon_{1} & -1 & \varepsilon_{1}+k_{d} \varepsilon_{2} & -\varepsilon_{2}
\end{array}\right]
$$

therefore,

$$
\begin{aligned}
& -\frac{1}{2}\left(A^{\top} P+P A\right)= \\
& \frac{1}{2} \underbrace{\left[\begin{array}{cccc}
\varepsilon_{1} k_{p}^{\prime} & -\varepsilon_{2} k_{i} & \varepsilon_{1}\left(k_{d}-k_{p}^{\prime}\right) & 0 \\
-\varepsilon_{2} k_{i} & \varepsilon_{1}(b-1)-2 \frac{\varepsilon_{2} k_{i}}{\varepsilon_{1}} & \varepsilon_{2} k_{i}-\varepsilon_{1} a & 0 \\
\varepsilon_{1}\left(k_{d}-k_{p}^{\prime}\right) & \varepsilon_{2} k_{i}-\varepsilon_{1} a & k_{d}\left(\frac{a}{b}-\varepsilon_{1}\right) & 0 \\
0 & 0 & 0 & \varepsilon_{2}
\end{array}\right]}_{Q_{1}}+
\end{aligned}
$$

$$
\frac{1}{2} \underbrace{\left[\begin{array}{cccc}
\varepsilon_{1} k_{p}^{\prime} & 0 & 0 & -\varepsilon_{2} k_{p}^{\prime} \\
0 & \varepsilon_{1}(b-1) & 0 & 0 \\
0 & 0 & k_{d}\left(\frac{a}{b}-\varepsilon_{1}\right) & -k_{d} \varepsilon_{2} \\
-\varepsilon_{2} k_{p}^{\prime} & 0 & -k_{d} \varepsilon_{2} & \varepsilon_{2}
\end{array}\right]}_{Q_{2}}
$$

The matrix $Q_{1}$ in (20) is positive definite if each element in its main diagonal is positive and the matrix is strictly diagonal-dominant. The matrix $Q_{2}$ is also positive definite for sufficiently small values of $\varepsilon_{1}$ and $\varepsilon_{2}$. Hence, we conclude that

$$
-\frac{1}{2}\left(A^{\top} P+P A\right)=\frac{1}{2}\left(Q_{1}+Q_{2}\right)=Q, \quad Q=Q^{\top}>0 .
$$

Computing the total time derivative of $V_{1}\left(\zeta_{1}\right)$ we obtain

$$
\dot{V}_{1}=-\zeta_{1}^{\top} Q \zeta_{1}+\zeta_{1}^{\top} P B\left(T_{e}-T_{e}^{*}\right) .
$$

To prove input-to-state stability with input $e_{x}$ let $q_{m}$ be the smallest eigenvalue of $Q$ and let $\gamma_{1} \geq|P B|$ then,

$$
\dot{V}_{1} \leq-q_{m}\left|\zeta_{1}\right|^{2}+\gamma_{1}\left|\zeta_{1}\right|\left|T_{e}-T_{e}^{*}\right|
$$

Next, we use (9) to obtain

$$
\begin{aligned}
\dot{V}_{1} \leq & -q_{m}\left|\zeta_{1}\right|^{2}+\frac{\gamma_{1} k_{M}}{2}\left|\zeta_{1}\right|\left[\left|e_{x}\right|^{2}+2\left|x^{*}\right|\left|e_{x}\right|\right] \\
\leq & -q_{m}\left|\zeta_{1}\right|^{2}+\frac{\gamma_{1} k_{M}}{4}\left[\frac{\left|e_{x}\right|^{4}}{\mu_{2}}+\mu_{2}\left|\zeta_{1}\right|^{2}\right] \\
& +\frac{\gamma_{1} k_{M}}{4}\left[\frac{4\left|x^{*}\right|^{2}\left|e_{x}\right|^{2}}{\mu_{1}}+\mu_{1}\left|\zeta_{1}\right|^{2}\right] \\
\leq & -\left[q_{m}-\frac{\gamma_{1} k_{M}}{4}\left(\mu_{1}+\mu_{2}\right)\right]\left|\zeta_{1}\right|^{2} \\
& +\frac{\gamma_{1} k_{M}}{4}\left[\frac{\left|e_{x}\right|^{2}}{\mu_{2}}+\frac{4\left|x^{*}\right|^{2}}{\mu_{1}}\right]\left|e_{x}\right|^{2} .
\end{aligned}
$$

For sufficiently small values of $\mu_{1}$ and $\mu_{2}$ the coefficient of $-\left|\zeta_{1}\right|^{2}$ is positive. The second term is a function of class $\mathcal{K}_{\infty}$ of $\left|e_{x}\right|$. In particular, we have $\zeta_{1} \rightarrow 0$ as $\mathrm{e}_{x} \rightarrow 0$.

Proof of Claim 2. Consider the function

$$
V_{2}\left(e_{x}\right)=\left|e_{x}\right|^{2},
$$

whose total time derivative along the trajectories of (19), yields

$\dot{V}_{2}=-2\left(R+k_{p x}\right) e_{x}^{\top} L^{-1} e_{x}-2 e_{x}^{\top} L^{-1} K x e_{w}-2 e_{w} e_{x}^{\top} \Delta$.

Using (2) we see that

$l_{m} \dot{V}_{2} \leq-\left(\frac{l_{m}}{l_{M}}\left(R+k_{p x}\right)-k_{M}|x|^{2}-|\Delta|^{2}\right)\left|e_{x}\right|^{2}+\left(k_{M}+l_{m}\right) e_{w}^{2}$

which is negative definite if $e_{w}=0$ and

$$
k_{p x} \geq-R+\left[k_{p x}^{\prime}+k_{M}|x|^{2}+|\Delta|^{2}\right]\left(l_{M} / l_{m}\right),
$$

which is coherent with (16). Hence, $V_{2}$ is an ISS-Lyapunov function and the system is input-to-state stable.

To finish the proof of Proposition 1, redefine $Q$ by removing the second term in the diagonal of $Q_{2}$ in (20). Note that the conditions for positive definiteness of $Q$ do 
not change, only its lower bound, which we now denote by $q_{m}^{\prime}$. Then, provided that we enforce $k_{p x}$ to

$k_{p x}:=-R+\left(k_{p x}^{\prime}+k_{M}|x|^{2}+|\Delta|^{2}+\frac{\gamma_{1} k_{M}}{4}\left[\frac{\left|e_{x}\right|^{2}}{\mu_{1}}+\frac{4\left|x^{*}\right|^{2}}{\mu_{2}}\right]\right) \frac{l_{M}}{l_{m}}$

which is also compatible with (16), we obtain

$$
\begin{gathered}
\dot{V}_{1}+\dot{V}_{2} \leq-k_{p x}^{\prime}\left|e_{x}\right|^{2}-\left[\frac{\varepsilon_{1}(b-1)}{2}-\frac{k_{M}}{l_{m}}-1\right] e_{w}^{2} \\
-\left[q_{m}^{\prime}-\frac{\gamma_{1} k_{M}}{4}\left(\mu_{1}+2 \mu_{2}\right)\right]\left|\zeta_{1}\right|^{2}
\end{gathered}
$$

which is negative semidefinite for small values of $\mu_{1}, \mu_{2}$ and large values of $b$-see the appendix for details.

Moreover, in view of the positivity of $P$, for appropriate (large) values of the control gains $a, b, k_{p}, k_{d}, k_{p x}$ and small values of $k_{i}, \varepsilon_{1}$ and $\varepsilon_{2}$ there exist positive constants $q_{1}, q_{2}$, $q_{3}$ such that

$$
\begin{gathered}
q_{1}\left(\left|e_{x}\right|^{2}+\left|\zeta_{1}\right|^{2}\right) \leq V_{1}\left(\zeta_{1}\right)+V_{2}\left(e_{x}\right) \leq q_{2}\left(\left|e_{x}\right|^{2}+\left|\zeta_{1}\right|^{2}\right) \\
\dot{V}_{1}\left(\zeta_{1}\right)+\dot{V}_{2}\left(e_{x}\right) \leq-q_{3}\left(\left|e_{x}\right|^{2}+\left|\zeta_{1}\right|^{2}\right) .
\end{gathered}
$$

Global exponential stability of the origin follows.

\section{Simulation Results}

We have tested our main result in simulations using SIMULINK $^{\mathrm{TM}}$ of MATLAB ${ }^{\mathrm{TM}}$. The parameters of the motors are $R=5, l_{0}=0.030 H, J=0.01 k g-m^{2}$ and $N_{r}=4$ while the controller gains are $k_{p}=1050, k_{i}=5 \mathrm{e}-4, k_{d}=1000$, $a=1500, b=3200$ and $k_{p x}^{\prime}=50$. The boundaries for $K_{j}(\theta), L_{j}(\theta),|P B|$ and $Q$ were fixed to $k_{M}=0.085$, $l_{m}=0.01, \gamma_{1}=105$ and $q_{m}^{\prime}=1 \mathrm{e}-5$ respectively. Finally, the constants $\mu_{1}=3 \mathrm{e}-6, \mu_{2}=1 \mathrm{e}-6, \varepsilon_{1}=0.04$ and $\varepsilon_{2}=1 \mathrm{e}-$ 5 were defined. Note that $P$ and $Q$ are positive definite for these values and $\dot{V}=\dot{V}_{1}+\dot{V}_{2}$ is negative definite.

The evaluation scenario consists in imposing a smooth signal reference constructed using the following function

$$
\omega^{*}(t)=\left(\frac{\omega_{f}^{*}-\omega_{0}^{*}}{2}\right)(\tanh (t-T)+1)+\omega_{0}^{*},
$$

The reference is obtained as the sum of three different implementations of the function above where $T=$ $4,14,25, \omega_{0}^{*}=0[\mathrm{rad} / \mathrm{s}]$, in the three cases, while $\omega_{f}^{*}=$ $100,-200,100[\mathrm{rad} / \mathrm{s}]$ and the initial conditions of the motor are equal to zero. The load torque is $T_{L}=1[\mathrm{Nm}]$ during the evaluation.

Under the conditions described above, the speed response of the closed-loop system is shown in Figure 2 where both the reference and actual speed are superimposed. Note the remarkable tracking achieved in spite of the sign inversion for the desired speed. Some chattering is exhibited by the motor speed, as the depicted in the sub-figure. This behavior is normal due to the commutation among the phases and corresponds to the situation when one of the phases requires larger values than the other, as it may be appreciated in Figure 3 and Figure 4. In this sense, the advantage of using the torque sharing approach is verified by the reduced magnitude of this chattering.

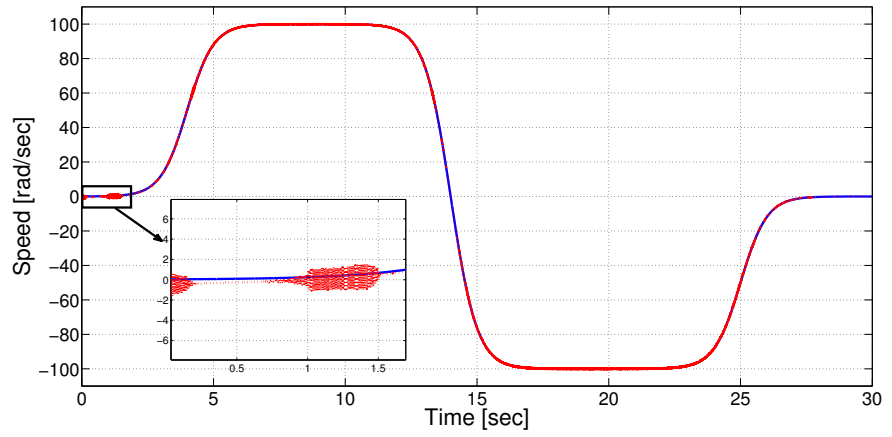

Fig. 2. Rotor angular velocity (red-line) and its reference (blue-line)

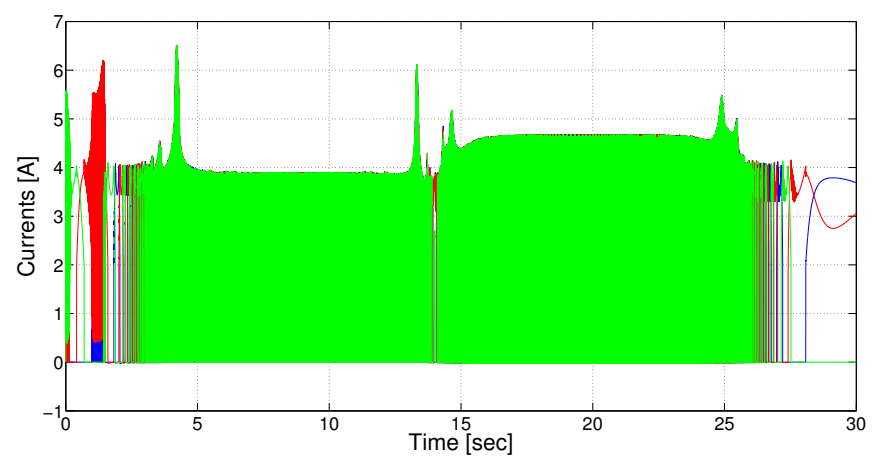

Fig. 3. Motor currents

With the aim to further illustrate the good performance of the proposed controller, in Figure 3 and Figure 4 are depicted the currents and the voltages required during the motor operation. In these figures it is important to note that the maximum values achieved by these signals (peaks of $6[\mathrm{~A}]$ for currents and peaks of 200[V] for voltages) are quite reasonable from an implementation point of view considering the load torque imposed to the motor.

Concerning the commutation of the excitations applied to the three phases, in Figure 5 and Figure 6 both the currents and the voltages are shown for an expanded period of time. In these figures, besides the illustration of the correct sequence application, it can be noticed how this sequence change when the speed crosses the zero value.

Finally and in order to illustrate the current tracking properties of the proposed controller, in Figure 7 are shown both the desired and actual current for one of the motor phases. Even that perfect tracking is not achieved this behavior is acceptable with respect to the speed performance. A better current response can be achieved by changing the controller gains (in particular increasing the value of $k_{p x}^{\prime}$ ) at the expense of having larger values for the voltages.

\section{CONCLUDING REMARKS}

As far as we know, we have presented the first controller guaranteeing global exponential stability for switchedreluctance motors without velocity measurements. Our main 


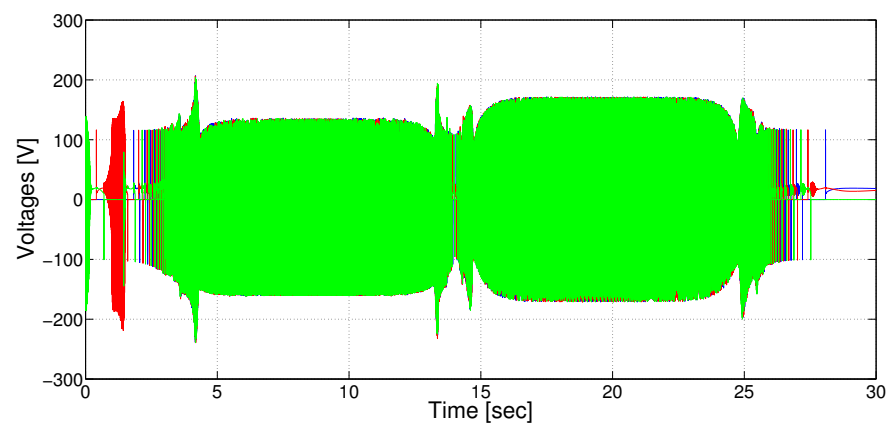

Fig. 4. Motor voltages

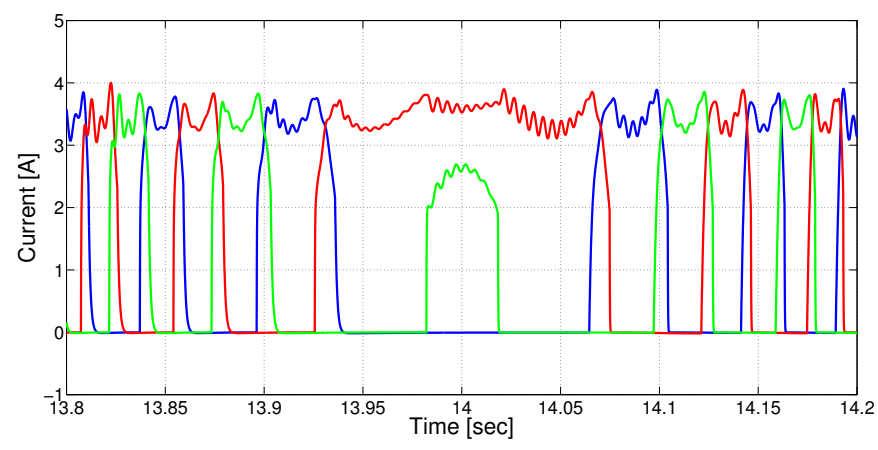

Fig. 5. Motor currents commutation

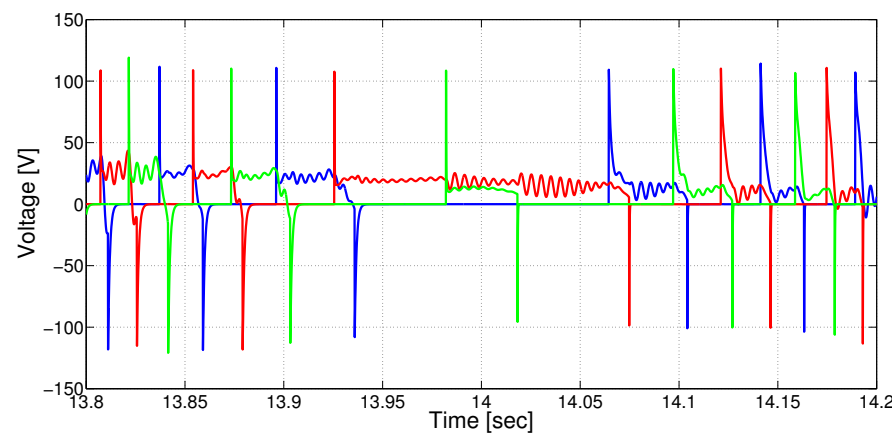

Fig. 6. Motor voltages commutation

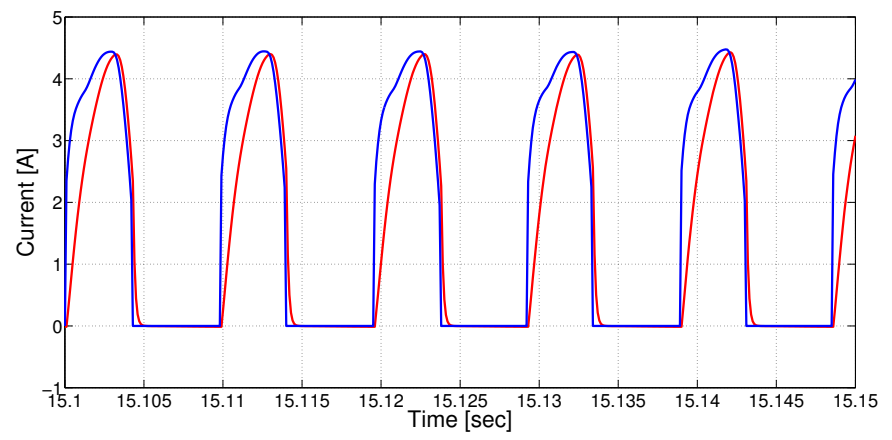

Fig. 7. Motor current (red-line) and its reference (blue-line) result is a preliminary step towards the open problem of theoretically-validated sensorless control for switchedreluctance motors. Current research is being carried out in this direction, in particular it focusses on the design of an angular-position observer to be implemented with a certaintyequivalence controller.

\section{Acknowledgements}

The work of G. Espinosa is supported by DGAPA-UNAM under grant IN111211. This work was mostly carried out while A. Loria was visiting FI-UNAM supported by the Programa de Posgrado en Ingeniería UNAM. A. Loria's and E. Chumacero's research leading to these results has also received funding from the European Union Seventh Framework Program [FP7/2007-2013] under grant agreement $\mathrm{n}^{\circ} 257462$ HYCON2 Network of excellence. E. Chumacero benefits from a scholarship by CONACyT, Mexico.

\section{REFERENCES}

[1] D. Taylor, "Pulse-width modulated control of electromechanical systems," IEEE Trans. Automat. Contr., vol. AC-37, pp. 524-528, 1992.

[2] M. Montanari, S. Peresada, and A. Tilli, "A speed-sensorless indirect field-oriented control for induction motors based on high gain speed estimation," Automatica, vol. 42, pp. 1637-1650, 2006.

[3] R. Marino, P. Tomei, and C. M. Verrelli, "A global tracking control for speed-sensorless induction motors," Automatica, vol. 40, pp. 10711077, 2004.

[4] R. Marino, P. Tomei, and C. M. Verrelli, "A nonlinear tracking control for sensorless induction motors," Automatica, vol. 41, pp. 1071-1077, 2005.

[5] G. Espinosa-Perez, P. Maya-Ortiz, M. Velasco-Villa, and H. SiraRamirez, "Passivity-based control of switched reluctance motors with nonlinear magnetic circuits," Control Systems Technology, IEEE Transactions on, vol. 12, pp. 439 - 448, may 2004.

[6] C. Hudson, N. Lobo, and R. Krishnan, "Sensorless control of single switch-based switched reluctance motor drive using neural network," Industrial Electronics, IEEE Transactions on, vol. 55, pp. 321 -329, jan. 2008.

[7] Y. Zheng, H. Sun, Y. Dong, and Z. Lei, "The research of stator current oriented method of switched reluctance motor," in Control and Decision Conference, 2008. CCDC 2008. Chinese, pp. $3184-3188$, july 2008 .

[8] W. Xiaoyuan, P. Hao, and G. Zhi, "Double switch reluctance motors synchronization control system," in Control and Decision Conference, 2008. CCDC 2008. Chinese, pp. $3754-3756$, july 2008.

[9] H. Peyrl, G. Papafotiou, and M. Morari, "Model predictive torque control of a switched reluctance motor," in Industrial Technology, 2009. ICIT 2009. IEEE International Conference on, pp. $1-6$, feb. 2009.

[10] L. dos Reis, F. Sobreira, A. Coelho, O. Almeida, J. Campos, and S. Daher, "Identification and adaptive speed control for switched reluctance motor using dsp," in Power Electronics Conference, 2009. COBEP '09. Brazilian, pp. 836 -841, 27 2009-oct. 12009.

[11] R. Ortega, A. Loría and R. Kelly, "A semiglobally stable output feedback PI2D regulator for robot manipulators," IEEE Trans. on Automat. Contr., vol. 40, no. 8, pp. 1432-1436, 1995.

[12] R. Krishnan, Switched Reluctance Motor Drives. CRC Press, 2001.

[13] M. Ilic-Spong, R. Marino, S. Peresada, and D. Taylor, "Feedback linearizing control of switched reluctance motors," IEEE Trans. Automat. Contr., vol. AC-32, pp. 371-379, 1987. 